Original Article

\title{
Comparison of muscle strength between hemodialysis patients and non-dialysis patients with chronic kidney disease
}

\author{
Nobuyuki Shirai, RPT, MSc ${ }^{1,2)}$, Suguru Yamamoto, MD²*, Yutaka Osawa, MD ${ }^{3)}$, \\ Atsuhiro Tsubaki, PhD, RPT ${ }^{4}$, Shinichiro Morishita, PhD, RPT ${ }^{5}$, \\ KANAMI IGARASHI, RPT, MSc ${ }^{1)}$, ICHIEI NARITA, MD ${ }^{2)}$ \\ 1) Department of Rehabilitation, Niigata Rinko Hospital, Japan \\ 2) Division of Clinical Nephrology and Rheumatology, Niigata University Graduate School of Medical \\ and Dental Sciences: 1-757 Asahimachi-dori, Niigata, Niigata 951-8510, Japan \\ 3) Internal Medicine, Niigata Rinko Hospital, Japan \\ 4) Institute for Human Movement and Medical Sciences, Niigata University of Health and Welfare, \\ Japan \\ 5) Department of Physical Therapy, School of Health Science, Fukushima Medical University, Japan
}

\begin{abstract}
Purpose] Muscle weakness in patients with chronic kidney disease is associated with several diseaserelated factors, and this study aimed to examine whether hemodialysis is one of risk factors for muscle weakness in patients with chronic kidney disease. [Participants and Methods] We conducted a cross-sectional study with 74 non-dialysis and 84 hemodialysis patients. Muscle strength evaluations were performed by measuring isometric knee extensor muscle strength and grip strength. Each evaluation item was compared between the hemodialysis and non-dialysis groups, and multiple regression analysis was performed to determine the factors associated with muscle strength. In addition, the correlation between lower-extremity muscle strength and grip strength was examined in each group. [Results] Isometric knee extensor muscle strength was significantly lower in the hemodialysis group than in the non-dialysis group. Grip strength was also significantly lower in the hemodialysis group than in the non-dialysis group. Hemodialysis was determined to be an independent risk factor associated with lower limb muscle strength as well as grip strength. The positive correlation between isometric knee extensor muscle strength and grip strength was almost the same in the groups. [Conclusion] Hemodialysis treatment was an independent risk factor for muscle weakness. Regular monitoring of grip strength may facilitate better management with physical therapy in hemodialysis patients.

Key words: Chronic kidney disease, Muscle strength decrease, Hemodialysis
\end{abstract}

(This article was submitted May 27, 2021, and was accepted Jul. 26, 2021)

\section{INTRODUCTION}

Sarcopenia, frailty, and protein-energy wasting (PEW) induce impairment in activities of daily living in patients with chronic kidney disease (CKD). The European Working Group on Sarcopenia in Older People defined sarcopenia as a muscle disease (muscle failure) rooted in adverse muscle changes that accrue during a lifetime, and suggested that attention should be focused on low muscle strength as a key characteristic of sarcopenia ${ }^{1)}$. CKD is one of the major risk factors for sarcopenia and frailty ${ }^{2,3)}$, which are probably due to not only traditional and CKD-specific risk factors but also dialysis-related factors ${ }^{4}$. However, few studies have directly compared muscle strength between CKD patients undergoing hemodialysis (HD) and

*Corresponding author. Suguru Yamamoto (E-mail: yamamots@med.niigata-u.ac.jp)

(C2021 The Society of Physical Therapy Science. Published by IPEC Inc.

(c) (1) $\odot$ This is an open-access article distributed under the terms of the Creative Commons Attribution Non-Commercial No Deriva-




CKD patients not receiving dialysis treatment. In addition, although grip strength is known to be associated with lower-limb muscle strength in the general population ${ }^{5}$, no study has compared this association between HD patients and non-dialysis patients.

In this study, we assessed and compared muscle strength between HD patients and non-dialysis CKD patients using two parameters: lower-extremity muscle strength and grip strength. In addition, the association of lower-limb muscle strength with upper-limb strength was examined and compared between HD and non-HD patients.

\section{PARTICIPANTS AND METHODS}

This was a cross-sectional study conducted at a single center. We recruited all outpatients undergoing HD at the CKD clinics of Niigata Rinko Hospital from January 2017 to March 2019. All patients were not undergoing exercise therapy. We excluded patients with difficulties in walking, those with histories of severe respiratory and cardiovascular diseases (because some evaluations impose a burden on cardiopulmonary function), and those who had been undergoing HD for $<1$ year. The background characteristics of all patients were retrospectively investigated using medical records. The following basic information was recorded: age, gender, body weight, body mass index, dry weight, serum albumin level, blood hemoglobin level, estimated glomerular filtration rate for non-HD patients, dialysis duration for HD patients, primary disease of CKD, and presence of comorbidities (including cerebrovascular disease, cardiovascular disease, diabetes, diabetic retinopathy, and diabetic nephropathy). Muscle strength was measured by a physical therapist at the Department of Rehabilitation of Niigata Rinko Hospital.

The protocol of this study complied with the Helsinki Declaration of 1975, as revised in 2013. This study was conducted with the approval of the Ethics Committee of Niigata Rinko Hospital (approval no. 3011-74) and registered at the University Hospital Medical Information Network Center (UMIN000035838). All patients provided written informed consent.

Isometric knee extensor muscle strength was measured using a handheld dynamometer ( $\mu$ Tas F-1 isometric myodynamometer; ANIMA Corp. Tokyo, Japan). The patients were instructed to sit upright and to look directly forward, with the torso vertical and the arms crossed in front of the chest. A pillow was placed under the knees, with the hip and knee joints at a $90^{\circ}$ angle. The length of the belt was adjusted so that the lower leg was vertical, and the belt was wrapped around the upright support of the test plinth. Moreover, a secure band was wound around the thighs to prevent compensatory movement of the hip joint. A sensor pad was placed approximately $5 \mathrm{~cm}$ from the tip of the lateral malleolus and was lightly supported during the measurements to prevent deviation. Furthermore, during the measurements, the patients were instructed not to hold their breath but to let the air out slowly and continuously. After a pre-test was performed to confirm the direction of movement, isometric knee extensor muscle strength with maximum effort for $5 \mathrm{~s}$ was measured twice on the left and right sides. The measurement from the side that showed a higher strength level was adopted and the average value. We analyzed the data adjusted for body weight (percent body weight [\%BW] $)^{6,7)}$.

Grip strength was measured using a digital dynamometer (Jamar Plus; Patterson Medical Ltd. Chicago, IL, USA). The patients were instructed to sit upright, and the height of the test plinth was adjusted such that the hip, knee, and ankle joints were at right angles. Thereafter, the patients were instructed to look directly forward and to maintain the torso in an upright position. The grip strength dynamometer was gripped with the shoulders in a neutral position, the elbow flexed at $90^{\circ}$, and the forearm in a neutral position. The patients were instructed not to hold their breath but to let the air out slowly and continuously during the measurements. Thereafter, maximum grip contraction was performed for $5 \mathrm{~s}$. Measurements were performed twice each on the left and right sides, and the average value $(\mathrm{kg})$ from the side with the higher strength level was adopted ${ }^{8}$.

The Short Physical Performance Battery (SPPB) is a simple evaluation of the physical capacity of the lower limbs. It involves three types of balance tests, a 4-m walk test, and a sit-to-stand test in which the time to rise from a chair five times is recorded. The results of these tests are scored from 0 to 4 and evaluated based on a total score of $0-12$ for all items. A low score indicates poor performance of the lower limbs. The usual gait speed was also evaluated in the present study, separately from the SPPB score ${ }^{9}$.

Frailty was evaluated based on the Japanese version of the Cardiovascular Health Study (J-CHS) criteria consisting of five components: weight loss, exhaustion, low physical activity, slowness, and weakness ${ }^{10)}$. (1) Weight loss was evaluated using the question, "Have you unintentionally lost 2 or more $\mathrm{kg}$ in the past 6 months?". (2) Exhaustion was measured using the question, "In the past 2 weeks, have you felt tired without a reason?". (3) Low physical activity was measured using two questions: "Do you engage in moderate levels of physical exercise or sports aimed at health?" and "Do you engage in low levels of physical exercise aimed at health?". (4) Slowness was measured using usual gait speed, which was determined by asking the patients to walk $4 \mathrm{~m}$ at a comfortable pace with the use of any walking aid to maintain balance and function. (5) Weakness was evaluated by measuring handgrip strength in the sitting position. The J-CHS criteria were scored as follows: (1) weight loss=1 point for a "yes" answer to the question; (2) exhaustion=1 point for a "yes" answer to the question; (3) low physical activity=1 point for a "no" answer to both questions; (4) slowness=1 point if gait speed was $<1.0 \mathrm{~m} / \mathrm{s}$; and (5) weakness $=1$ point if handgrip strength was $<28 \mathrm{~kg}$ in male and $<18 \mathrm{~kg}$ in female. We calculated the total J-CHS score by summing up the scores of the components. Frail, pre-frail, and non-frail were defined as meeting the criteria for 3-5, 1-2, and 0 components, respectively. In addition, those who scored 0 points for physical activity were defined as having an exercise habit. 
Each measurement value is represented as the median (interquartile range). The Mann-Whitney U test and $\chi^{2}$ test were performed for the comparison of the evaluation items between the HD and non-HD groups. We also performed multiple regression analysis forced input method with isometric knee extensor muscle strength and grip strength as dependent variables. The independent variables were age, gender, diabetes mellitus, cardiac disease, cerebrovascular disease, serum albumin levels, and exercise habits, which are potential confounders according to previous studies ${ }^{11,12)}$, as well as HD treatment, which is the main variable in our research question. To investigate the relationship between lower-extremity muscle strength and grip strength in both the HD and non-HD groups, Spearman's rank correlation coefficient test was performed. IBM SPSS Statistics (version 27, Stats Guild Inc, Chiba, Japan) was used for the analysis, and the significance level was set to 5\%.

\section{RESULTS}

This study enrolled 169 patients with CKD stage 3a-5D. Among them, 11 patients did not meet the inclusion criteria. Table 1 shows the clinical characteristics of the patients in the HD and non-HD groups. The median duration of HD treatment was 6.0 (interquartile range 2.0-11.0) years among HD patients, and the estimated glomerular filtration rate was 39.1 (27.1-50.9) $\mathrm{mL} / \mathrm{min} / 1.73 \mathrm{~m}^{2}$ among non-HD patients. The number of patients according to CKD stage was 31 (stage $3 \mathrm{a}$ ), 21 (stage 3b), 17 (stage 4), and 5 (stage 5). Patients in the HD group had lower body weight, lower body mass index, lower albumin level, and lower hemoglobin level than those in the non-HD group. In addition, the HD group included more patients with cerebrovascular disease, heart disease, diabetic nephropathy, and frailty. With respect to the primary disease of CKD,

Table 1. Clinical characteristics in the non-dialysis CKD group and HD group

\begin{tabular}{|c|c|c|c|}
\hline & $\begin{array}{c}\text { Non-dialysis CKD } \\
(\mathrm{n}=74)\end{array}$ & $\begin{array}{c}\mathrm{HD} \\
(\mathrm{n}=84)\end{array}$ & p-value \\
\hline Age (years) & $75.0(66.0-81.0)$ & $73.0(65.3-79.0)$ & 0.420 \\
\hline Male, n (\%) & $50(67.6)$ & $50(59.5)$ & 0.300 \\
\hline Weight or DW (kg) & $61.9(53.2-69.2)$ & $52.6(44.6-60.3)$ & $<0.001$ \\
\hline BMI $\left(\mathrm{kg} / \mathrm{m}^{2}\right.$ or $\left.\mathrm{DW} / \mathrm{m}^{2}\right)$ & $24.0(22.5-26.1)$ & $21.5(18.7-24.7)$ & $<0.001$ \\
\hline Dialysis duration (years) & & $6.0(2.0-11.0)$ & \\
\hline \multicolumn{4}{|l|}{ Comorbid conditions } \\
\hline Cerebrovascular disease, $\mathrm{n}(\%)$ & $6(8.1)$ & $29(34.5)$ & $<0.001$ \\
\hline Cardiac disease, $\mathrm{n}(\%)$ & $18(24.3)$ & $70(83.3)$ & $<0.001$ \\
\hline Diabetes mellitus, n (\%) & $51(68.9)$ & $57(67.9)$ & 1.0 \\
\hline Diabetic retinopathy, n (\%) & $19(25.7)$ & $24(28.6)$ & 0.720 \\
\hline Diabetic nephropathy, n (\%) & $12(16.2)$ & $31(36.9)$ & $<0.01$ \\
\hline \multicolumn{4}{|l|}{ Primary kidney disease } \\
\hline Diabetic nephropathy, n (\%) & $31(41.9)$ & $31(37.0)$ & 0.620 \\
\hline Glomerulonephritis, n (\%) & $5(6.8)$ & $30(35.7)$ & $<0.001$ \\
\hline Hypertension, n (\%) & $26(35.1)$ & $13(15.5)$ & 0.005 \\
\hline Other nephropathies, n (\%) & $10(13.5)$ & $10(11.9)$ & 0.810 \\
\hline J-CHS (score) & $1.0(0-2.0)$ & $3.0(2.0-3.0)$ & $<0.001$ \\
\hline Frail, n (\%) & $11(14.9)$ & $46(54.8)$ & $<0.001$ \\
\hline Pre-frail, n (\%) & $37(0.5)$ & $31(36.9)$ & 0.070 \\
\hline Non-frail, n (\%) & $26(35.1)$ & $7(8.3)$ & $<0.001$ \\
\hline Exercise habit, n (\%) & $42(56.8)$ & $27(32.1)$ & 0.002 \\
\hline \multicolumn{4}{|l|}{ Laboratory values } \\
\hline eGFR $\left(\mathrm{mL} / \mathrm{min} / 1.73 \mathrm{~m}^{2}\right)$ & $39.1(27.1-50.9)$ & & \\
\hline Albumin (g/dL) & $3.9(3.7-4.2)$ & $3.4(3.2-3.6)$ & $<0.001$ \\
\hline Hemoglobin (g/dL) & $12.9(11.6-14.4)$ & $11.2(10.5-12.4)$ & $<0.001$ \\
\hline \multicolumn{4}{|l|}{ Physical function } \\
\hline Lower-limb muscle strength (\%BW) & $50.2(35.0-59.1)$ & $30.1(24.2-39.0)$ & $<0.001$ \\
\hline Grip strength $(\mathrm{kg})$ & $30.1(19.6-38.6)$ & $20.0(15.6-25.1)$ & $<0.001$ \\
\hline SPPB (score) & $12.0(11.0-12.0)$ & $9.5(6.3-11.0)$ & $<0.001$ \\
\hline Usual gait speed $(\mathrm{m} / \mathrm{s})$ & $1.2(1.0-1.3)$ & $1.0(0.7-1.1)$ & $<0.001$ \\
\hline
\end{tabular}

BMI: body mass index; \%BW: percent body weight; CKD: chronic kidney disease; DW: dry weight; eGFR: estimated glomerular filtration rate; HD: hemodialysis; J-CHS: Japanese version of the Cardiovascular Health Study; SPPB: Short Physical Performance Battery. 
chronic glomerulonephritis and hypertension were significantly more common in the HD group. Isometric knee extensor muscle strength was significantly lower in the HD group than in the non-HD group (HD: $30.1 \% \mathrm{BW}$ [24.2\%BW-39.0\%BW] vs. non-HD: $50.2 \% \mathrm{BW}$ [35.0\%BW-59.1\%BW], $\mathrm{p}<0.001)$. Table 2 shows the results of multiple regression analysis of factors related to lower limb muscle strength. As a result, $\mathrm{HD}\left(R^{2}=0.43, \beta=-0.31, \mathrm{p}=0.001\right)$ was extracted as a factor related to isometric knee extensor muscle strength. Grip strength was also significantly lower in the HD group than in the non-HD group (HD: 20.0 [15.6-25.1] kg vs. non-HD: 30.1 [19.6-38.6] kg, p<0.001). Table 2 shows the results of multiple regression analysis of factors related to grip strength. As a result, $\operatorname{HD}\left(R^{2}=0.65, \beta=-0.28, \mathrm{p}<0.001\right)$ was extracted as a factor related to grip strength. In the analysis of the correlation between isometric knee extensor muscle strength and grip strength in the two groups, a positive correlation was found in the HD group $(r=0.60, \mathrm{p}<0.001)$ and an almost similar trend was observed in the non-HD group $(r=0.65, \mathrm{p}<0.001)$

\section{DISCUSSION}

In this study, we demonstrated that lower-extremity muscle strength and grip strength were poorer in HD patients than in non-dialysis CKD patients. Multiple regression analysis showed that HD treatment was one of the strongest factors associated with muscle strength impairment. In addition, lower-extremity muscle strength and grip strength were positively correlated in HD patients, with an almost identical trend observed in non-HD patients.

Frailty, sarcopenia, and PEW are more frequently observed in CKD patients, and their severity increases with deterioration of kidney function in non-dialysis patients with $\mathrm{CKD}^{13)}$. The frequency of frailty and sarcopenia in HD patients is also higher than that in patients with normal kidney function, which is considered to be associated with mortality ${ }^{14,15)}$. Muscle strength greatly affects sarcopenia and frailty; however, no reports comparing muscle strength between HD patients and non-dialysis CKD patients have been published to date. In this study, we found that lower-extremity muscle strength and grip strength were lower in patients undergoing HD than in non-dialysis CKD patients. We adjusted leg muscle strength to account for body weight, and the results suggested that patients undergoing HD had lower muscle quality and lower muscle mass than those not undergoing HD.

In multivariate analysis, lower-limb muscle strength was affected by age, gender, diabetes, exercise habit, and albumin level, in addition to HD treatment. Grip strength was affected by HD treatment, age, gender, exercise habit, and albumin level. In general, muscle strength decreases with age ${ }^{16}$, and it has been clarified that females are weaker than male ${ }^{17}$. In addition, CKD patients are known to have increased proteolysis due to decreased dietary intake, nutrient loss due to HD treatment, decreased protein synthesis, and increased catabolism ${ }^{15}$. In other words, continued malnutrition reduces energy storage sources such as protein, muscle, and fat, which can easily lead to PEW and sarcopenia ${ }^{4}$. Diabetic neuropathy affects motor function, and it has been reported that diabetes itself reduces muscle strength per unit muscle mass ${ }^{18)}$. Previous studies on exercise habits and strength reported that patients with type 2 diabetes with $>150$ min of exercise in a week had higher lower-limb muscle strength than those without an exercise habit, and that physical activity time and lower-limb muscle strength were correlated ${ }^{19)}$. Our results in CKD patients showed the same trend as those in previous studies, and HD treatment was an important factor associated with lower-limb muscle strength and grip strength (Table 2).

End-stage CKD and/or dialysis-related factors are believed to induce impairment of muscle strength. For example, accumulation of uremic toxins including indoxyl sulfate, which are difficult to remove with conventional dialysis treatment, causes metabolic abnormalities in muscle cells and leads to muscle atrophy ${ }^{20)}$. Elevated parathyroid hormone levels ${ }^{21)}$ and abnormal vitamin D metabolism ${ }^{22}$ ) have also been reported to be associated with decreased muscle mass in animal models. Fluid retention and anemia in HD patients induce an increase in cardiac output ${ }^{23}$. Muscle disuse due to long durations of bed rest during dialysis sessions and post-treatment fatigue may be associated with muscle atrophy in HD patients. Additionally, it is well known that CKD patients undergoing HD frequently develop depression. The reported prevalence of depression in the dialysis population ranges from $22.8 \%$ to $39.3 \%{ }^{24)}$, and depression may lead to less physical activity. On the basis of the above findings, a more effective approach to kidney disease or dialysis therapy will be needed to maintain or improve muscle strength in HD patients.

Physicians should accurately evaluate muscle strength in HD patients during daily treatment in the clinic. As many di-

Table 2. Relationship between muscle strength and HD

\begin{tabular}{lcccc}
\hline & \multicolumn{3}{c}{ Unadjusted model } & \multicolumn{2}{c}{${\text { Adjusted } \text { model }^{*}}^{$\cline { 2 - 5 }$}$} & $\beta(95 \% \mathrm{CI})$ & $\mathrm{p}$-value & $\beta(95 \% \mathrm{CI})$ & $\mathrm{p}$-value \\
\hline Lower limb muscle strength & $-0.498(-20.698$ to -11.759$)$ & $<0.001$ & $-0.305(-15.566$ to -4.320$)$ & $0.001^{\mathrm{a}}$ \\
Grip strengh & $-0.474(-12.313$ to -6.727$)$ & $<0.001$ & $-0.281(-8.360$ to -2.925$)$ & $<0.001^{\mathrm{b}}$ \\
\hline
\end{tabular}

95\% CI: 95\% Confidence Interval.

*Adjusted for age, gender, diabetes mellitus, cardiac disease, cerebrovascular disease, serum albumin levels, exercise habits.

aadjusted $R^{2}=0.425$.

badjusted $R^{2}=0.646$. 
alysis facilities do not have a physiotherapist, a muscle strength evaluation method that can be easily performed by other health-care professionals is needed. In addition, it has been reported that the frequency of falls increases in the general elderly population with impaired lower-limb extension muscle strength ${ }^{25)}$. Therefore, lower-limb muscle strength measurement is important for preventing falls and frailty also in HD patients. Grip strength is known to be associated with leg muscle strength and walking speed in the general population ${ }^{5}$. In this study, a positive correlation between lower-extremity muscle strength and grip strength was observed in HD patients, and an almost similar trend was observed in the non-dialysis group. Thus, the assessment of grip strength can predict systemic muscle strength in patients undergoing HD in the same manner as in the general population. In this study, the HD group was composed of elderly patients (Table 1). Even in this population, grip strength can be a good parameter for assessing total muscle strength.

To improve the activities of daily living or quality of life and increase the chances of survival of HD patients, it may be important to perform appropriate rehabilitation starting from the non-dialysis CKD stage. A previous study assessed the effect of exercise intervention consisting of daily walking for $30 \mathrm{~min}$ or 8,000-10,000 steps and resistance training at least three times per week for 12 months in non-HD patients with CKD. As a result, grip strength and knee extensor muscle strength significantly improved without affecting kidney function ${ }^{26}$. In addition, when patients with CKD stage $3 \mathrm{~b}$ or 4 underwent resistance training of the lower limbs (three sets, 10-12 repetitions) for 8 weeks, improvements in knee extension muscle strength, muscle mass, and exercise capacity were observed ${ }^{27}$. For the above reasons, exercise intervention from the early (non-dialysis) stage of CKD seems necessary for maintaining physical function during maintenance dialysis treatment, as well as for proper CKD and dialysis management.

This study had several limitations. This was a cross-sectional study that did not clarify the causal relationship between HD and impaired muscle strength. Although we found that HD is one of the strongest factors affecting muscle strength in CKD patients, there might be other factors associated with muscle strength. Physical activity, which is known to affect muscle strength, was investigated as the presence of an exercise habit, not as an objective measure of activity. This cross-sectional study was performed in a small number of patients at a single center. To achieve better clinical outcomes, large-scale interventional prospective studies are needed to better understand the importance of muscle strength in HD patients.

In conclusions, isometric knee extensor muscle strength and grip strength are lower in HD patients than in non-dialysis CKD patients. HD treatment is one of the independent factors affecting impaired muscle strength in CKD patients. A clear correlation exists between upper- and lower-limb muscle strength in HD patients, similar to non-HD patients. Regular assessment of grip strength can be performed to determine systemic muscle strength in HD patients.

\section{Conflicts of interest}

All authors have no conflicts of interest to declare.

\section{ACKNOWLEDGMENT}

The authors acknowledge the expert comments and suggestions from Kou Kitabayashi.

\section{REFERENCES}

1) Cruz-Jentoft AJ, Bahat G, Bauer J, et al. Writing Group for the European Working Group on Sarcopenia in Older People 2 (EWGSOP2), and the Extended Group for EWGSOP2: Sarcopenia: revised European consensus on definition and diagnosis. Age Ageing, 2019, 48: 16-31. [Medline] [CrossRef]

2) Foley RN, Wang C, Ishani A, et al.: Kidney function and sarcopenia in the United States general population: NHANES III. Am J Nephrol, 2007, 27: 279-286. [Medline] [CrossRef]

3) Reese PP, Cappola AR, Shults J, et al. CRIC Study Investigators: Physical performance and frailty in chronic kidney disease. Am J Nephrol, 2013, 38: 307-315. [Medline] [CrossRef]

4) Fouque D, Kalantar-Zadeh K, Kopple J, et al.: A proposed nomenclature and diagnostic criteria for protein-energy wasting in acute and chronic kidney disease. Kidney Int, 2008, 73: 391-398. [Medline] [CrossRef]

5) Rantanen T, Guralnik JM, Foley D, et al.: Midlife hand grip strength as a predictor of old age disability. JAMA, 1999, 281: 558-560. [Medline] [CrossRef]

6) Matsuzawa R, Matsunaga A, Wang G, et al.: Relationship between lower extremity muscle strength and all-cause mortality in Japanese patients undergoing dialysis. Phys Ther, 2014, 94: 947-956. [Medline] [CrossRef]

7) Hansen EM, McCartney CN, Sweeney RS, et al.: Hand-held dynamometer positioning impacts discomfort during quadriceps strength testing: a validity and reliability study. Int J Sports Phys Ther, 2015, 10: 62-68. [Medline]

8) Erkol İnal E, Çarlı AB, Çanak S, et al.: Effects of hyperthyroidism on hand grip strength and function. J Rehabil Res Dev, 2015, 52: 663-668. [Medline] [CrossRef]

9) Guralnik JM, Simonsick EM, Ferrucci L, et al.: A short physical performance battery assessing lower extremity function: association with self-reported disability and prediction of mortality and nursing home admission. J Gerontol, 1994, 49: M85-M94. [Medline] [CrossRef]

10) Satake S, Arai H: The revised Japanese version of the Cardiovascular Health Study criteria (revised J-CHS criteria). Geriatr Gerontol Int, 2020, 20: $992-993$. [Medline] [CrossRef]

11) Isoyama N, Qureshi AR, Avesani CM, et al.: Comparative associations of muscle mass and muscle strength with mortality in dialysis patients. Clin J Am Soc 
Nephrol, 2014, 9: 1720-1728. [Medline] [CrossRef]

12) Kim JC, Shapiro BB, Zhang M, et al.: Daily physical activity and physical function in adult maintenance hemodialysis patients. J Cachexia Sarcopenia Muscle, 2014, 5: 209-220. [Medline] [CrossRef]

13) Roshanravan B, Khatri M, Robinson-Cohen C, et al.: A prospective study of frailty in nephrology-referred patients with CKD. Am J Kidney Dis, 2012, 60: 912-921. [Medline] [CrossRef]

14) Johansen KL, Chertow GM, Jin C, et al.: Significance of frailty among dialysis patients. J Am Soc Nephrol, 2007, 18: 2960-2967. [Medline] [CrossRef]

15) Kim JK, Choi SR, Choi MJ, et al.: Prevalence of and factors associated with sarcopenia in elderly patients with end-stage renal disease. Clin Nutr, 2014, 33: 64-68. [Medline] [CrossRef]

16) Kamel HK: Sarcopenia and aging. Nutr Rev, 2003, 61: 157-167. [Medline] [CrossRef]

17) Izawa KP, Oka K, Watanabe S, et al.: Gender-related differences in clinical characteristics and physiological and psychosocial outcomes of Japanese patients at entry into phase II cardiac rehabilitation. J Rehabil Med, 2008, 40: 225-230. [Medline] [CrossRef]

18) Andersen H: Motor dysfunction in diabetes. Diabetes Metab Res Rev, 2012, 28: 89-92. [Medline] [CrossRef]

19) Shirai N, Tsubaki A, Morishita S, et al.: The association between time spent in performing physical activity and physical function in outpatients with type 2 diabetes who may have diabetic neuropathy. Diabetes Metab Syndr, 2020, 14: 2111-2116. [Medline] [CrossRef]

20) Sato E, Mori T, Mishima E, et al.: Metabolic alterations by indoxyl sulfate in skeletal muscle induce uremic sarcopenia in chronic kidney disease. Sci Rep, 2016, 6: 36618. [Medline] [CrossRef]

21) Thomas SS, Mitch WE: Parathyroid hormone stimulates adipose tissue browning: a pathway to muscle wasting. Curr Opin Clin Nutr Metab Care, 2017, 20: 153-157. [Medline] [CrossRef]

22) Dzik KP, Kaczor JJ: Mechanisms of vitamin D on skeletal muscle function: oxidative stress, energy metabolism and anabolic state. Eur J Appl Physiol, 2019, 119: 825-839. [Medline] [CrossRef]

23) Harada T, Obokata M, Kurosawa K, et al.: Relationships of high cardiac output with ventricular morphology, myocardial energetics, and energy costs in hemodialysis patients with preserved ejection fraction. Int J Cardiovasc Imaging, 2019, 35: 469-479. [Medline] [CrossRef]

24) King-Wing Ma T, Kam-Tao Li P: Depression in dialysis patients. Nephrology (Carlton), 2016, 21: 639-646. [Medline] [CrossRef]

25) Pijnappels M, van der Burg PJ, Reeves ND, et al.: Identification of elderly fallers by muscle strength measures. Eur J Appl Physiol, 2008, 102: 585-592. [Medline] [CrossRef]

26) Hiraki K, Shibagaki Y, Izawa KP, et al.: Effects of home-based exercise on pre-dialysis chronic kidney disease patients: a randomized pilot and feasibility trial. BMC Nephrol, 2017, 18: 198. [Medline] [CrossRef]

27) Watson EL, Greening NJ, Viana JL, et al.: Progressive resistance exercise training in CKD: a feasibility study. Am J Kidney Dis, 2015, 66: 249-257. [Medline] [CrossRef] 